\title{
HECT E3 Ligases: A Tale With Multiple Facets
}

\author{
Janine Weber ${ }^{1}$, Simona Polo ${ }^{1,2 *}$ and Elena Maspero ${ }^{1 *}$ \\ ${ }^{1}$ Fondazione Istituto FIRC di Oncologia Molecolare, Milan, Italy, ${ }^{2}$ Dipartimento di Oncologia ed Emato-Oncologia, Università \\ degli Studi di Milano, Milan, Italy
}

Ubiquitination plays a pivotal role in several cellular processes and is critical for protein degradation and signaling. E3 ubiquitin ligases are the matchmakers in the ubiquitination cascade, responsible for substrate recognition. In order to achieve selectivity and specificity on their substrates, HECT E3 enzymes are tightly regulated and exert their function in a spatially and temporally controlled fashion in the cells. These characteristics made HECT E3s intriguing targets in drug discovery in the context of cancer biology.

Keywords: ubiquitin, E3 ligase, cancer, inhibitor, HECT regulations

\section{HECT E3 LIGASES}

\section{OPEN ACCESS}

Edited by:

Julien Licchesi,

University of Bath, United Kingdom

Reviewed by:

Pierre G. Lutz,

Centre National de la Recherche

Scientifique (CNRS), France

Olivier Staub,

Université de Lausanne, Switzerland

${ }^{*}$ Correspondence:

Simona Polo

simona.polo@ifom.eu

Elena Maspero

elena.maspero@ifom.eu

Specialty section:

This article was submitted to

Integrative Physiology,

a section of the journal

Frontiers in Physiology

Received: 18 December 2018

Accepted: 18 March 2019

Published: 03 April 2019

Citation:

Weber J, Polo S and Maspero E (2019) HECT E3 Ligases: A Tale With

Multiple Facets.

Front. Physiol. 10:370.

doi: 10.3389/fphys.2019.00370
Post-translational modification of proteins by the addition of a ubiquitin (Ub) moiety can induce alteration in protein stability, function, activity, localization and interaction (Komander and Rape, 2012). This tightly regulated process (reviewed in Oh et al., 2018) requires the sequential action of a cascade of three enzymes: the Ub-activating enzyme (E1), Ub-conjugating enzymes (E2s), and Ub ligases (E3s). E3 ligases are the matchmakers of the enzymatic cascade, as they are capable of conferring a high degree of specificity and selectivity toward target substrates in cells. The human proteome codifies for more than $600 \mathrm{E} 3 \mathrm{~s}$ (Li et al., 2008; Berndsen and Wolberger, 2014) that can be divided into three classes: the largest class is the RING (Really Interesting New Gene/Ubox)-type E3s with about 600 members (reviewed in Metzger et al., 2014), followed by the HECT (Homologous to E6AP C-Terminus)-type E3s with about 28 members (reviewed in Rotin and Kumar, 2009 and Sluimer and Distel, 2018) and the RBR (RING between RING)-type E3s with about 14 members (reviewed in Dove and Klevit, 2017 and Reiter and Klevit, 2018). Whereas the RING E3 ligases function as allosteric activators of the E2 and scaffolds that bring the latter in close proximity to the substrate, the HECT and RBR E3 ligases catalyze substrate ubiquitination in a twostep reaction: in the first step, they accept the activated $\mathrm{Ub}$ from the $\mathrm{E} 2$ in a transthiolation reaction on their catalytic cysteine, and in the second step, the Ub moiety is transferred to a lysine on the target substrate.

In this review, we focus on the HECT-containing E3 ligases. Invariably at their C-terminus, all HECT E3s present the catalytic HECT domain, composed of a bulkier N-terminal lobe (N-lobe) that contains the $\mathrm{E} 2$ binding domain, and a C-terminal lobe (C-lobe) carrying the catalytic cysteine. The two lobes are connected by a flexible hinge region that allows the C-lobe to move around in order to facilitate the Ub transfer from the E2 to the E3 (Huang et al., 1999; Verdecia et al., 2003; Kamadurai et al., 2009).

According to the domain organization present in the N-terminal part of the proteins, the HECT E3s can be subdivided into three main families (Table 1; Rotin and Kumar, 2009; Scheffner and Kumar, 2014; Sluimer and Distel, 2018). The best characterized family is the NEDD4 family, consisting of nine human members: ITCH, SMURF1, SMURF2, WWP1, WWP2, NEDD4, NEDD4-2, HECW1, and HECW2. The NEDD4 members share similar domain structure and consist of a membrane/lipid-binding $\mathrm{C} 2$ domain, two to four WW domains for substrate 
recognition and a C-terminal HECT domain (Fajner et al., 2017). The second class, the HERC family, is characterized by one or more regulators of chromatin condensation 1 (RCC)-like domains (RLD), which serve as a guanine nucleotide exchange factor (GEF) for the small GTPase in membrane trafficking processes (Sanchez-Tena et al., 2016). This family consists of six members that can be subdivided into four 'small' and two 'large' HERCs, where the latter, HERC1 and HERC2, are the largest HECT E3s with about 5000 residues. The remaining 13 HECTs do not share specific domains at the $\mathrm{N}$-terminus and, for this reason, are classified as "other" HECT ligases (Scheffner and Kumar, 2014).

\section{REGULATION OF HECT E3 LIGASE ACTIVITY}

The activity of HECT E3s is tightly regulated in terms of chain specificity (mono- or poly-ubiquitination and Ub chain linkage), interaction with the $\mathrm{E} 2$ and recognition of the substrate.

TABLE 1 | Overview of the human HECT E3 ligase subfamilies with the respective members, including their domain organization.

\begin{tabular}{lll}
\hline Subfamily & Domains & Members \\
\hline NEDD4 & C2, WW (4x), HECT & NEDD4, NEDD4-2, ITCH, \\
& C2, WW (3x), HECT & SMURF2 \\
& C2, WW (2x), HECT & SMURF1, HECW1, HECW2 \\
HERC & RLD (2x), SPRY, WD40, HECT & HERC1 \\
& RLD (3x), Cytb5, MIB, ZnF, & HERC2 \\
& DOC, HECT & \\
& RLD, HECT & HERC3, HERC4, HERC5, \\
& HERC6 \\
"Other" HECT & AZUL, HECT & E6AP \\
& ARM, UBA, WWE, BH3, UBM, & HUWE1 \\
& HECT & \\
ANK, HECT & HACE1 \\
& ARM (2x), WWE, HECT & TRIP12 \\
& UBA, ZnF, PABC, HECT & UBR5 \\
& IQ, HECT & UBE3B, UBE3C \\
ANK, SUN, MIB, HECT & HECTD1 \\
HECT & HECTD2, HECTD4 \\
DOC, HECT & HECTD3 \\
PHD/RING, HECT & G2E3 \\
Filamin, HECT & AREL1 \\
&
\end{tabular}

Protein domains were predicted by the InterPro server (Finn et al., 2017). HECT E3 ligases are grouped into three subfamilies, NEDD4, HERC, and "other" HECT E3 ligases based on their domain architecture N-terminal to the HECT domain. Domain abbreviations used are as follows: C2, C2 domain $\mathrm{Ca}^{2+}$ binding domain); WW, WW domain; HECT, homologous to EGAP C-terminus; RLD, Regulator of Chromosome Condensation 1 repeat-like domain; SPRY, B30.2/SPRY domain; WD4O, W-D repeat domain; Cytb5, cytochrome b5-like heme/steroidbinding domain; DOC, APC10/DOC domain; MIB, MIB-HERC2 domain; ZnF, Zinc finger domain; AZUL, amino-terminal Zn-binding domain of UBE3A ligase; ARM, Armadillo repeat-containing domain; UBA, ubiquitin-associated domain; WWE, WWE domain; $B H 3, B C l-2$ homology 3 domain; UBM, ubiquitin-binding motif; ANK, Ankyrin repeat-containing domain; $P A B C$, polyadenylate-binding protein C-terminal domain; IQ, IQ motif/EF-hand binding site; SUN, SAD1/UNC domain; PHD, PHD-type zinc finger; Filamin, filamin/ABP280 repeat-like domain.
The ability to build linkage-specific poly-Ub chains appears to be an intrinsic feature of the HECT enzymes, as they are able to generate distinct Ub chains regardless of the paired E2 enzymes. NEDD4 family members primarily synthesize K63 chains (Kim and Huibregtse, 2009; Maspero et al., 2013; Kristariyanto et al., 2015), while E6AP is a K48-specific enzyme (Wang and Pickart, 2005; Kim and Huibregtse, 2009) and HUWE1 generates K6-, K11-, and K48- linked poly-Ub chains (Jackl et al., 2018). In most of the cases, the detailed mechanism through which they assemble specific poly-Ub chains remains unknown. In the case of NEDD4, the presence of a non-covalent Ub-binding site, called the Ub exosite, in the $\mathrm{N}$-lobe appears to be required for enzyme processivity, possibly by stabilizing and orienting the distal end of growing Ub chains on the substrate (Maspero et al., 2011, 2013).

Precise control of E3 ligase activity is needed to ensure that their functions are restricted until required. Several HECT E3s are kept in a catalytically inactive state by intramolecular interactions between the $\mathrm{N}$-terminal region (either the $\mathrm{C} 2$ or the linker region between the WW domains) and the C-terminal HECT domain (Wiesner et al., 2007; Mari et al., 2014; Riling et al., 2015; Chen et al., 2017; Zhu et al., 2017). For other E3s, such as E6AP and HUWE1, the mechanism is different but always requires intermolecular interactions. The crystal structure of the free HECT domain of E6AP suggests that it forms a trimer (Huang et al., 1999) and that the trimeric state activates the E3 ligase (Ronchi et al., 2014). In contrast, the C-terminal region of HUWE1 is maintained in an inactive conformation by homodimerization that occurs at the HECT domain. The engagement of the dimerization region by an activation segment located at the N-terminal of the protein seems to relieve this inhibitory mechanism (Sander et al., 2017).

A third level of regulation is represented by adaptor proteins that can modulate both the E2-E3 interaction and the interaction with the substrate. An example of the former is represented by SMAD7. SMURF1 and SMURF2 have low binding affinities for the E2-conjugating enzyme $\mathrm{UbcH7}$, providing a point of control for regulating the Ub ligase activity through the action of auxiliary proteins. Indeed, SMAD7, functioning as a bridge between the E2 and E3, stabilizes an active complex and promotes, thus, the ligase activity (Ogunjimi et al., 2005). In other cases, adaptor proteins may regulate the $\mathrm{E} 3$ ligase by promoting its engagement with the substrate. The most famous example is represented by the adaptor protein E6 that binds to a LxxLL motif of the E6AP HECT ligase and forms, together with E6AP, a binding surface for the p53 protein. Consequently, p53 becomes K48-polyubiquitinated and degraded by the $26 \mathrm{~S}$ proteasome (Huibregtse et al., 1993; Martinez-Zapien et al., 2016). NEDD4 family E3s usually recruit substrates via the WW domains that serve as direct binding sites for PPxY motifs present on the targets (Persaud et al., 2009). In this case, cooperation with auxiliary proteins confers the ability to cope with a larger number of substrates. Indeed, in the last decade, proteins such as ARTs in yeast and ARRDCs in mammals were found to modulate the ubiquitination of PY-negative substrates (Lin et al., 2008; Polo and Di Fiore, 2008; Mund and Pelham, 2009; Han et al., 2013). Other adaptor proteins which contribute to NEDD4 family members regulation are NDFIP1 and NDFIP2, transmembrane proteins that localize 
to Golgi, endosomes, and multivesicular bodies (Harvey et al., 2002; Shearwin-Whyatt et al., 2006). Through their cytoplasmic PY motifs they allow the association of NEDD4 family members to their specific substrates [e.g., DMT1 (Foot et al., 2008), ENaC (Konstas et al., 2002) the water channel AQP2 (Trimpert et al., 2017)] and directly modulate the activity of these E3s (Mund and Pelham, 2009, 2010).

Notably, HECTs themselves can function as adaptors for other conjugating enzymes as in the case of HERC2 whose binding to the N-terminal domain of E6AP increases the catalytic activity of E6AP (Kuhnle et al., 2011).

Finally, the catalytic activity of the HECT enzymes is often spatially and temporally regulated by post-translational modifications. Activating modifications can contribute to the release of auto-inhibiting conformational states of the E3s. For example, the phosphorylation of ITCH on the three residues of the proline-rich region releases the auto-inhibitory state generated by the binding of the $\mathrm{C} 2$ and the first WW domain to the HECT domain (Gallagher et al., 2006). Likewise, FGFR and EGFR activate NEDD4 by inducing a Src-dependent phosphorylation of specific tyrosine residues in the $\mathrm{C} 2$ and HECT domains, opening thus the closed conformation (Persaud et al., 2014); a mechanism that seems to be in place also for NEDD4-2 (Grimsey et al., 2018). With an opposite behavior, phosphorylation of a specific residue in the HECT domain of E6AP by the kinase c-Abl disrupts the trimeric state and therefore inhibits the ligase (Chan et al., 2013).

\section{HECT E3 LIGASES AND THEIR UNDEFINED ROLE IN TUMORIGENESIS}

Ubiquitin ligases regulate a wide range of cellular processes and are involved in many human pathologies. Abnormal expression or dysfunction of HECT E3s have been shown in many different cancers. The current knowledge often suggests a dual role for these ligases in tumorigenesis, which might depend on the tissue context and/or additional events that affect their activity. Here, we will review the recent literature on E6AP, NEDD4, and HUWE1, and highlight excellent reviews for additional reading (Bernassola et al., 2008; Rotin and Kumar, 2009; Scheffner and Kumar, 2014; Zou et al., 2015; Wang et al., 2017; Kao et al., 2018).

The classical example of an HECT associated with cancer is E6AP. Since its discovery in 1993, it was evident that E6AP drives human papilloma virus (HPV)-induced cervical carcinogenesis, exerting its activity toward the tumor suppressor p53 through its association with the viral protein E6. E6 is an adaptor protein of the HPV and it is capable of binding to the N-terminal of EA6P and the DNA-binding domain of p53 (Huibregtse et al., 1993; Scheffner et al., 1993; Beaudenon and Huibregtse, 2008), acting as an allosteric activator of E6AP (Mortensen et al., 2015), similarly to HERC2 that binds to the same region (Kuhnle et al., 2011). In addition to HPV-induced cancer, E6AP drives cancer progression in B-cell lymphoma where it degrades PML, allowing the tumor cells to bypass PML-induced senescence (Wolyniec et al., 2012). While E6AP appears to have a pro-oncogenic function, a few papers support a tumor-suppressive function for E6AP in breast and prostate cancers (Srinivasan and Nawaz, 2011; Levav-Cohen et al., 2012; Ramamoorthy et al., 2012; Mansour et al., 2016) and in non-small cell lung cancer where depletion of E6AP contributes to a decreased expression of the INK4/ARF locus (Gamell et al., 2017).

NEDD4 and NEDD4-2 E3s are instead modulators of endocytosis of several membrane proteins such as growth factor receptors [EGFR (Katz et al., 2002) and IGFR (Vecchione et al., 2003; Cao et al., 2008)] and ion channels [ENaC (Staub et al., 1996; Harvey et al., 2001), $\mathrm{Na}_{v}$ s (Fotia et al., 2004), and KCNQs (Ekberg et al., 2007; Jespersen et al., 2007)] therefore they are important players in the maintenance of cellular homeostasis. Mutations at the $\mathrm{C}$-terminal of $\mathrm{ENaC}$ subunits that abrogate the interaction with NEDD4-2 are the cause of Liddle's syndrome, an autosomal dominant disorder with severe sodium retention and hypertension (Staub et al., 1996).

Overexpression of NEDD4 has been reported in several cancer types and downregulation of NEDD4 appears to reduce proliferation, migration and invasion of cancer cells (reviewed in Zou et al., 2015). The relevance of NEDD4 in the tumorigenic process was initially associated with the identification of the tumor suppressor PTEN as a NEDD4 substrate (Trotman et al., 2007; Wang et al., 2007; Kim et al., 2008; Amodio et al., 2010). Later observations linked RAS activation to NEDD4 overexpression and subsequent PTEN degradation in human colorectal cancer (Zeng et al., 2014). However, studies conducted in NEDD4 knock out (KO) mice showed that PTEN stability was not affected by the E3 ligase deficiency (Fouladkou et al., 2008), while others showed that overexpression of NEDD4 in colorectal cancers promotes cancer cell growth independently of PTEN and $\mathrm{PI} 3 \mathrm{~K} / \mathrm{AKT}$ signaling, arguing that NEDD4-mediated regulation of PTEN is microenvironment and/or cell-type specific, and that other yet-unknown substrates are implicated in the process (Eide et al., 2013). While this latter remains an intriguing hypothesis, it is interesting to note that in vivo NEDD4 is reported to degrade many of its substrates, while in vitro its activity is clearly K63-specific (Kim and Huibregtse, 2009; Maspero et al., 2013). A possible explanation for this behavior resides in the involvement of adaptor proteins that could influence the specific type of Ub chains catalyzed by E3s [e.g., NUMB (Shao et al., 2017)] or deubiquitinases that may edit the Ub chains.

A last case study is represented by HUWE1, which is also frequently overexpressed in tumors (Chen et al., 2005; Confalonieri et al., 2009; Myant et al., 2017). Again, HUWE1 has been associated with both pro-oncogenic and tumor suppressor functions since it is responsible for K48-mediated degradation of a great variety of substrates ranging from the oncoprotein MYC (Zhao et al., 2008; Inoue et al., 2013; Myant et al., 2017) to the anti-apoptotic protein MCL1, (Zhong et al., 2005) to the tumor suppressor p53 (Chen et al., 2005) and BRCA1 (Wang et al., 2014). Particularly controversial is the role of HUWE1 in the regulation of MYC. On the one hand, HUWE1 is able to enhance tumor cell proliferation by K63-poly ubiquitination and activation of the transcription regulator MYC (Adhikary et al., 2005), on the other hand, depletion (Inoue et al., 2013) or mutation (Myant et al., 2017) of HUWE1 lead to increased MYC levels, thereby promoting skin and colon tumorigenesis. 
Clearly, a precise understanding of HUWE1 function in the various cancers relies heavily on the identification of its direct substrates and the type of Ub modification occurring to them.

\section{TARGET SITES AND SPECIFICITY OF HECT E3 LIGASE INHIBITORS}

As previously described, the regulatory mechanisms of HECT E3s are quite diverse and, therefore, provide a promising opportunity for drug discovery (Chen et al., 2018). Based on the actual knowledge, we can imagine different ways to inhibit their activity, namely: (i) by blocking the binding of the E2 enzymes or adaptor proteins; (ii) by tackling the catalytic cysteine of the enzymes; (iii) by targeting specific regulatory surfaces such as the Ub exosite; (iv) by impairing substrate recognition; and (v) by modulating the oligomeric state (Figure 1).
Molecules that block the HECT-E2 binding were found by Mund et al. (2014). By using a phage library, the authors isolated and modified bicyclic peptides that specifically bind to the HECT domains of SMURF2, NEDD4-1, WWP1, and HUWE1, competing with the E2 binding. Further improvement of the most promising peptide generated Heclin (HECT ligase inhibitor), a reversible inhibitor with a low micromolar affinity that, however, did not inhibit the E2 binding of the HECTs but rather caused a conformational change that renders their catalytic cysteine more susceptible to oxidation.

With the idea of identifying covalent modifiers of the catalytic cysteine of NEDD4, Kathman et al. (2015) found compounds that selectively react with a non-catalytic cysteine present in the Ub exosite of NEDD4 and NEDD4-2. Interestingly, no inhibition was observed for the NEDD4 family member WWP1 that also contains a cysteine in close proximity to the one seen in NEDD4, or for E6AP that does not contain

\section{A}

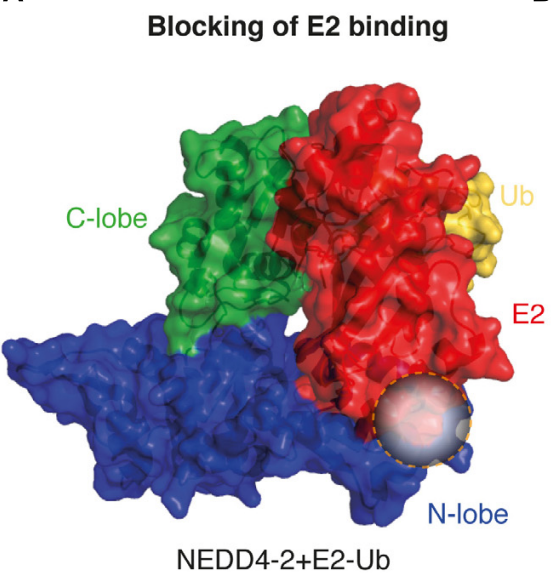

C

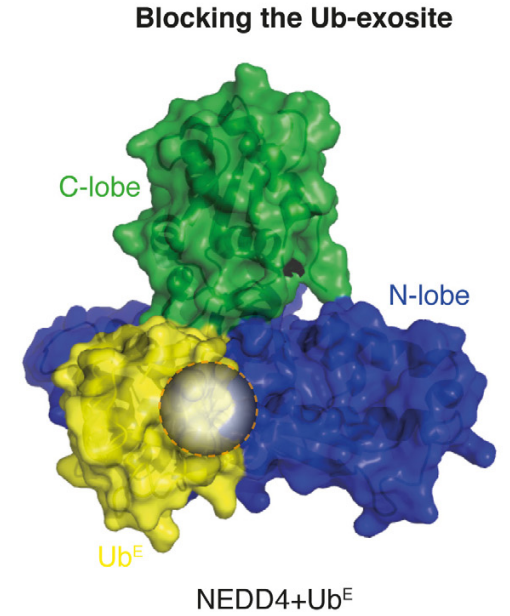

B

D

\section{Inhibiting the catalytic cysteine}

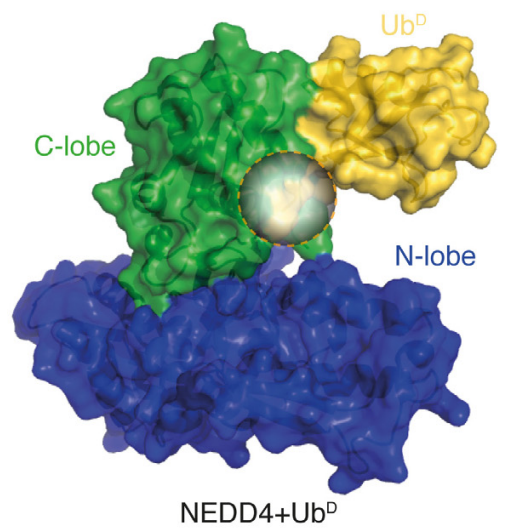

Inhibiting oligomerization

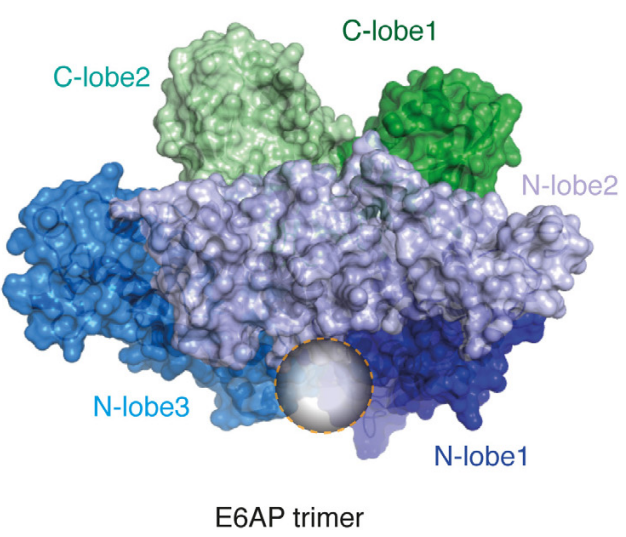

FIGURE 1 | Predicted inhibitory targets for HECT E3s activity. Surface representations of known crystal structures of HECT E3s. In circles are highlighted the possible binding sites for small molecules. (A) Blocking the E2 binding: HECTNEDD4-2 in complex with Ub (gold)-loaded E2 (red; PDB 3JVZ). (B) Inhibition of the catalytic cysteine: Ub (Ub , gold)-loaded HECT ${ }^{N E D D 4}$ complex; PDB 4BBN. (C) Blocking the Ub exosite: HECTNEDD4 in complex with Ub bound to the exosite (UbE, yellow; PDB 4BBN). (D) Inhibition of the oligomerization: HECTE6AP trimeric complex (1D5F). C-lobes and N-lobes are depicted in green and blue, respectively. 
a cysteine in this region (Kathman et al., 2015). Another compound that may bind to the $\mathrm{Ub}$ exosite of the HECT domain is $\mathrm{I} 3 \mathrm{C}$ (1H-indol-3-yl-carbinol), a phytochemical found in cruciferous vegetables that has an antiproliferative effect in cancers (Ahmad et al., 2010). I3C was found to interact with NEDD4 in vitro at micromolar concentrations (Adhikary et al., 2005). Through in silico binding simulations between I3C and the NEDD4 crystal structure, I3C was predicted to bind to the hydrophobic pocket of the N-lobe near the $\mathrm{Ub}$ exosite. In a follow-up study, Quirit et al. (2017) overcame the low binding affinity of I3C by screening a small library of $N$-benzyl or $N$-phenyl I3C analogs and identified 1-benzyl-indole-3-carbinol (1-benzyl-I3C) as a more potent inhibitor. However, the binding mode and the specificity of this compound has not been experimentally validated.

A recent approach suggests the use of specific Ub mutants identified by phage display (Ub variant, $\mathrm{UbV}$ ) to modulate HECT catalysis (Zhang et al., 2016). The screen performed against 19 of the 28 human HECT enzymes lead to the identification of variants that are capable to bind the N-lobe exosite but also the N-lobe surface involved in the interaction with the E2 (Zhang et al., 2016). Binding of these variants promote inhibition or activation depending on the E3 tested and the type of modifications present in the UbV underlying the complexity of the catalytic mechanism in place. While a generalization of the process is impossible, these reagents appear to be interesting tools for further studies.

A few molecules have been found to inhibit the HECTs, impairing substrate binding. An in silico screening of the hydrophobic pocket of the WW domains of SMURF1 led to the identification of compounds that possess features similar to the PPXY motif. These compounds bind the ligase and block SMAD1 ubiquitination, possibly disrupting the WW domain:SMAD1 interaction (Okada et al., 2009; Kato et al., 2011; Cao et al., 2014). However, affinity, binding mode and selectivity remain to be tested.

For many small molecules and inhibitors, the binding sites, the mechanism or the specificity have not been determined. By a high-throughput screening of small molecules, Eilers and coworkers identified two compounds that selectively inhibit the enzyme activity of HUWE1, as seen by a reduced substrate ubiquitination. Both compounds were found to inhibit the ligase activity with $\mathrm{IC}_{50}$ values in the low micromolar range, leaving NEDD4 family members or the E1 and E2 enzymes unaffected. The compounds reduced the growth of colorectal cancer cells, but not that of HUWE1-depleted or normal epithelial cells of the colon (Peter et al., 2014). However, how these compounds work on HUWE1 remains unknown (Peter et al., 2014). With a similar approach, Rossi and co-workers identified several putative ITCH inhibitors, including Clomipramine, an FDAapproved drug that is used in the clinic to treat psychiatric disorders. Clomipramine and its analogs specifically block the HECT catalytic activity of the NEDD4 family member ITCH but not that of the RING ligase Ring1B (Rossi et al., 2014). The authors clarified the mode of action of this class of drugs, showing that it specifically inhibits the transthioesterification reaction (the transfer of Ub from the E2 to the HECT domain), implying some common features at the level of the HECT members (Rossi et al., 2014).

\section{CONCLUDING REMARKS}

Although tackling the ubiquitination system rather than the proteasome seems to be a promising avenue for therapeutic drug discovery, targeting HECT E3s to manipulate their activity is challenging for several reasons. First of all, we still lack the complete picture of their ubiquitome and their mechanism of action. Which substrates do HECT E3s ubiquitinate? What impact does ubiquitination have on their function proteolytic or non-proteolytic? How are these substrates recognized and how is their ubiquitination regulated in time and space and in different cellular conditions? What are the mechanisms the different HECT E3s apply to ubiquitinate their targets? So far, we only have a few answers for a small number of ligases and substrates due to the fact that ubiquitination is a dynamic and highly regulated process, and that the interaction with substrates is often transient with a low binding affinity. Besides the PPxY motif that is recognized by the WW domains of NEDD4 family members, no other substrate binding motif is known. An additional challenge is represented by redundancy. While E3s target multiple substrates, a specific substrate may be modulated by several E3s, depending also on the cell context. The high conservation of the HECT domain within the HECT family makes it a difficult target for which to develop specific inhibitory compounds. Finally, most of the HECTs act as both tumor suppressors and oncogenes, and more information is needed in order to find specific and effective compounds. Thus, acquiring more insights into the structural composition and the ubiquitination mechanism used by the different HECT E3s is of paramount importance in order to open new avenues for therapeutic interventions.

\section{AUTHOR CONTRIBUTIONS}

JW, EM, and SP conceptualized and wrote this review. JW prepared the figure. All authors approved the final version of the manuscript and agreed to be accountable for the content of the work.

\section{FUNDING}

Work in SP's laboratory was supported by the Associazione Italiana per la Ricerca sul Cancro (AIRC-IG\#IG19875) and the Italian Ministry of Education, Universities and Research (PRIN 20152CB22L). EM was supported by CARIPLO (2017-0746).

\section{ACKNOWLEDGMENTS}

We thank Wessen Maruwge for critically reading and editing the manuscript. 


\section{REFERENCES}

Adhikary, S., Marinoni, F., Hock, A., Hulleman, E., Popov, N., Beier, R., et al. (2005). The ubiquitin ligase HectH9 regulates transcriptional activation by Myc and is essential for tumor cell proliferation. Cell 123, 409-421. doi: 10.1016/j. cell.2005.08.016

Ahmad, A., Sakr, W. A., and Rahman, K. M. (2010). Anticancer properties of indole compounds: mechanism of apoptosis induction and role in chemotherapy. Curr. Drug Targets 11, 652-666. doi: 10.2174/138945010791170923

Amodio, N., Scrima, M., Palaia, L., Salman, A. N., Quintiero, A., Franco, R., et al. (2010). Oncogenic role of the E3 ubiquitin ligase NEDD4-1, a PTEN negative regulator, in non-small-cell lung carcinomas. Am. J. Pathol. 177, 2622-2634. doi: 10.2353/ajpath.2010.091075

Beaudenon, S., and Huibregtse, J. M. (2008). HPV E6, E6AP and cervical cancer. BMC Biochem. 9(Suppl. 1):S4. doi: 10.1186/1471-2091-9-S1-S4

Bernassola, F., Karin, M., Ciechanover, A., and Melino, G. (2008). The HECT family of E3 ubiquitin ligases: multiple players in cancer development. Cancer Cell 14, 10-21. doi: 10.1016/j.ccr.2008.06.001

Berndsen, C. E., and Wolberger, C. (2014). New insights into ubiquitin E3 ligase mechanism. Nat. Struct. Mol. Biol. 21, 301-307. doi: $10.1038 / \mathrm{nsmb} .2780$

Cao, X. R., Lill, N. L., Boase, N., Shi, P. P., Croucher, D. R., Shan, H. B., et al. (2008). Nedd 4 controls animal growth by regulating IGF-1 signaling. Sci. Signal. 1:ra5. doi: 10.1126/scisignal.1160940

Cao, Y., Wang, C., Zhang, X., Xing, G., Lu, K., Gu, Y., et al. (2014). Selective small molecule compounds increase BMP-2 responsiveness by inhibiting Smurf1-mediated Smad1/5 degradation. Sci. Rep. 4:4965 doi: 10.1038/srep04965

Chan, A. L., Grossman, T., Zuckerman, V., Campigli Di Giammartino, D., Moshel, O., Scheffner, M., et al. (2013). c-Abl phosphorylates E6AP and regulates its E3 ubiquitin ligase activity. Biochemistry 52, 3119-3129. doi: 10. 1021/bi301710c

Chen, D., Gehringer, M., and Lorenz, S. (2018). Developing small-molecule inhibitors of HECT-type ubiquitin ligases for therapeutic applications: challenges and opportunities. Chembiochem 19, 2123-2135. doi: 10.1002/cbic. 201800321

Chen, D. L., Kon, N., Li, M. Y., Zhang, W. Z., Qin, J., and Gu, W. (2005). ARF-BP1/mule is a critical mediator of the ARF tumor suppressor. Cell 121, 1071-1083. doi: 10.1016/j.cell.2005.03.037

Chen, Z., Jiang, H., Xu, W., Li, X., Dempsey, D. R., Zhang, X., et al. (2017). A tunable brake for HECT ubiquitin ligases. Mol. Cell 66, 345.e6-357.e6. doi: 10.1016/j.molcel.2017.03.020

Confalonieri, S., Quarto, M., Goisis, G., Nuciforo, P., Donzelli, M., Jodice, G., et al. (2009). Alterations of ubiquitin ligases in human cancer and their association with the natural history of the tumor. Oncogene 28, 2959-2968. doi: 10.1038/ onc. 2009.156

Dove, K. K., and Klevit, R. E. (2017). RING-between-RING E3 ligases: emerging themes amid the variations. J. Mol. Biol. 429, 3363-3375. doi: 10.1016/j.jmb. 2017.08.008

Eide, P. W., Cekaite, L., Danielsen, S. A., Eilertsen, I. A., Kjenseth, A., Fykerud, T. A., et al. (2013). NEDD4 is overexpressed in colorectal cancer and promotes colonic cell growth independently of the PI3K/PTEN/AKT pathway. Cell Signal 25, 12-18. doi: 10.1016/j.cellsig.2012.08.012

Ekberg, J., Schuetz, F., Boase, N. A., Conroy, S. J., Manning, J., Kumar, S., et al. (2007). Regulation of the voltage-gated $\mathrm{K}(+)$ channels KCNQ2/3 and KCNQ3/5 by ubiquitination. Novel role for Nedd4-2. J. Biol. Chem. 282, 12135-12142. doi: 10.1074/jbc.M609385200

Fajner, V., Maspero, E., and Polo, S. (2017). Targeting HECT-type E3 ligases insights from catalysis, regulation and inhibitors. FEBS Lett. 591, 2636-2647. doi: 10.1002/1873-3468.12775

Finn, R. D., Attwood, T. K., Babbitt, P. C., Bateman, A., Bork, P., Bridge, A. J., et al. (2017). InterPro in 2017-beyond protein family and domain annotations. Nucleic Acids Res. 45, D190-D199. doi: 10.1093/nar/gkw1107

Foot, N. J., Dalton, H. E., Shearwin-Whyatt, L. M., Dorstyn, L., Tan, S. S., Yang, B., et al. (2008). Regulation of the divalent metal ion transporter DMT1 and iron homeostasis by a ubiquitin-dependent mechanism involving Ndfips and WWP2. Blood 112, 4268-4275. doi: 10.1182/blood-2008-04-15 0953
Fotia, A. B., Ekberg, J., Adams, D. J., Cook, D. I., Poronnik, P., and Kumar, S. (2004). Regulation of neuronal voltage-gated sodium channels by the ubiquitinprotein ligases Nedd4 and Nedd4-2. J. Biol. Chem. 279, 28930-28935. doi: 10.1074/jbc.M402820200

Fouladkou, F., Landry, T., Kawabe, H., Neeb, A., Lu, C., Brose, N., et al. (2008). The ubiquitin ligase Nedd4-1 is dispensable for the regulation of PTEN stability and localization. Proc. Natl. Acad. Sci. U.S.A. 105, 8585-8590. doi: 10.1073/pnas. 0803233105

Gallagher, E., Gao, M., Liu, Y. C., and Karin, M. (2006). Activation of the E3 ubiquitin ligase Itch through a phosphorylation-induced conformational change. Proc. Natl. Acad. Sci. U.S.A. 103, 1717-1722. doi: 10.1073/pnas. 0510664103

Gamell, C., Gulati, T., Levav-Cohen, Y., Young, R. J., Do, H., Pilling, P., et al. (2017). Reduced abundance of the E3 ubiquitin ligase E6AP contributes to decreased expression of the INK4/ARF locus in non-small cell lung cancer. Sci. Signal 10:eaaf8223. doi: 10.1126/scisignal.aaf8223 doi: 10.1126/scisignal. aaf8223

Grimsey, N. J., Narala, R., Rada, C. C., Mehta, S., Stephens, B. S., Kufareva, I., et al. (2018). A tyrosine switch on NEDD4-2 E3 ligase transmits GPCR inflammatory signaling. Cell Rep. 24, 3312.e5-3323.e5. doi: 10.1016/j.celrep.2018.08.061

Han, S. O., Kommaddi, R. P., and Shenoy, S. K. (2013). Distinct roles for betaarrestin2 and arrestin-domain-containing proteins in beta2 adrenergic receptor trafficking. EMBO Rep. 14, 164-171. doi: 10.1038/embor.2012.187

Harvey, K. F., Dinudom, A., Cook, D. I., and Kumar, S. (2001). The Nedd4-like protein KIAA0439 is a potential regulator of the epithelial sodium channel. J. Biol. Chem. 276, 8597-8601. doi: 10.1074/jbc.C000906200

Harvey, K. F., Shearwin-Whyatt, L. M., Fotia, A., Parton, R. G., and Kumar, S. (2002). N4WBP5, a potential target for ubiquitination by the Nedd4 family of proteins, is a novel Golgi-associated protein. J. Biol. Chem. 277, 9307-9317. doi: 10.1074/jbc.M110443200

Huang, L., Kinnucan, E., Wang, G., Beaudenon, S., Howley, P. M., Huibregtse, J. M., et al. (1999). Structure of an E6AP-UbcH7 complex: insights into ubiquitination by the E2-E3 enzyme cascade. Science 286, 1321-1326. doi: 10. 1126/science.286.5443.1321

Huibregtse, J. M., Scheffner, M., and Howley, P. M. (1993). Localization of the E6-AP regions that direct human papillomavirus E6 binding, association with p53, and ubiquitination of associated proteins. Mol. Cell Biol. 13, 4918-4927. doi: 10.1128/MCB.13.8.4918

Inoue, S., Hao, Z. Y., Elia, A. J., Cescon, D., Zhou, L., Silvester, J., et al. (2013). Mule/Huwe1/Arf-BP1 suppresses Ras-driven tumorigenesis by preventing c-Myc/Miz1-mediated down-regulation of p21 and p15. Genes Dev. 27, 11011114. doi: $10.1101 / \operatorname{gad} .214577 .113$

Jackl, M., Stollmaier, C., Strohaker, T., Hyz, K., Maspero, E., Polo, S., et al. (2018). beta-sheet augmentation is a conserved mechanism of priming HECT E3 ligases for ubiquitin ligation. J. Mol. Biol. 430(18 Pt B), 3218-3233. doi: 10.1016/j.jmb. 2018.06.044

Jespersen, T., Membrez, M., Nicolas, C. S., Pitard, B., Staub, O., Olesen, S. P., et al. (2007). The KCNQ1 potassium channel is down-regulated by ubiquitylating enzymes of the Nedd4/Nedd4-like family. Cardiovasc. Res. 74, 64-74. doi: 10. 1016/j.cardiores.2007.01.008

Kamadurai, H. B., Souphron, J., Scott, D. C., Duda, D. M., Miller, D. J., Stringer, D. et al. (2009). Insights into ubiauitin transfer cascades from a structure of a UbcH5B similar to Ubiauitin-HECTNEDD4L complex. Mol. Cell 36, 10951102. doi: 10.1016/j.molcel.2009.11.010

Kao, S. H., Wu, H. T., and Wu, K. J. (2018). Ubiquitination by HUWE1 in tumorigenesis and beyond. J. Biomed. Sci. 25:67. doi: 10.1186/s12929-0180470-0

Kathman, S. G., Span, I., Smith, A. T., Xu, Z., Zhan, J., Rosenzweig, A. C., et al. (2015). A small molecule that switches a ubiquitin ligase from a processive to a distributive enzymatic mechanism. J. Am. Chem. Soc. 137, 12442-12445. doi: 10.1021/jacs.5b06839

Kato, S., Sangadala, S., Tomita, K., Titus, L., and Boden, S. D. (2011) A synthetic compound that potentiates bone morphogenetic protein-2-induced transdifferentiation of myoblasts into the osteoblastic phenotype. Mol. Cell. Biochem. 349, 97-106. doi: 10.1007/s11010-010-0664-6

Katz, M., Shtiegman, K., Tal-Or, P., Yakir, L., Mosesson, Y., Harari, D., et al. (2002). Ligand-independent degradation of epidermal growth factor receptor involves 
receptor ubiquitylation and Hgs, an adaptor whose ubiquitin-interacting motif targets ubiquitylation by Nedd4. Traffic 3, 740-751. doi: 10.1034/j.1600-0854. 2002.31006.x

Kim, H. C., and Huibregtse, J. M. (2009). Polyubiquitination by HECT E3s and the determinants of chain type specificity. Mol. Cell Biol. 29, 3307-3318. doi: 10.1128/MCB.00240-09

Kim, S. S., Yoo, N. J., Jeong, E. G., Kim, M. S., and Lee, S. H. (2008). Expression of NEDD4-1, a PTEN regulator, in gastric and colorectal carcinomas. APMIS 116, 779-784. doi: 10.1111/j.1600-0463.2008.00999.x

Komander, D., and Rape, M. (2012). The ubiquitin code. Annu. Rev. Biochem. 81, 203-229. doi: 10.1146/annurev-biochem-060310-170328

Konstas, A. A., Shearwin-Whyatt, L. M., Fotia, A. B., Degger, B., Riccardi, D., Cook, D. I., et al. (2002). Regulation of the epithelial sodium channel by N4WBP5A, a novel Nedd4/Nedd4-2-interacting protein. J. Biol. Chem. 277, 29406-29416. doi: 10.1074/jbc.M203018200

Kristariyanto, Y. A., Choi, S. Y., Rehman, S. A., Ritorto, M. S., Campbell, D. G., Morrice, N. A., et al. (2015). Assembly and structure of Lys33linked polyubiquitin reveals distinct conformations. Biochem. J. 467, 345-352. doi: 10.1042/BJ20141502

Kuhnle, S., Kogel, U., Glockzin, S., Marquardt, A., Ciechanover, A., Matentzoglu, K., et al. (2011). Physical and functional interaction of the HECT ubiquitin-protein ligases E6AP and HERC2. J. Biol. Chem. 286, 19410-19416. doi: 10.1074/jbc.M110.205211

Levav-Cohen, Y., Wolyniec, K., Alsheich-Bartok, O., Chan, A. L., Woods, S. J., Jiang, Y. H., et al. (2012). E6AP is required for replicative and oncogeneinduced senescence in mouse embryo fibroblasts. Oncogene 31, 2199-2209. doi: 10.1038/onc.2011.402

Li, W., Bengtson, M. H., Ulbrich, A., Matsuda, A., Reddy, V. A., Orth, A., et al. (2008). Genome-wide and functional annotation of human E3 ubiquitin ligases identifies MULAN, a mitochondrial E3 that regulates the organelle's dynamics and signaling. PLoS One 3:e1487. doi: 10.1371/journal.pone.0001487

Lin, C. H., MacGurn, J. A., Chu, T., Stefan, C. J., and Emr, S. D. (2008). Arrestinrelated ubiquitin-ligase adaptors regulate endocytosis and protein turnover at the cell surface. Cell 135, 714-725. doi: 10.1016/j.cell.2008.09.025

Mansour, M., Haupt, S., Chan, A. L., Godde, N., Rizzitelli, A., Loi, S., et al. (2016). The E3-ligase E6AP represses breast cancer metastasis via regulation of ECT2Rho signaling. Cancer Res. 76, 4236-4248. doi: 10.1158/0008-5472.CAN-151553

Mari, S., Ruetalo, N., Maspero, E., Stoffregen, M. C., Pasqualato, S., Polo, S., et al. (2014). Structural and functional framework for the autoinhibition of Nedd4-family ubiquitin ligases. Structure 22, 1639-1649. doi: 10.1016/j.str.2014.09.006

Martinez-Zapien, D., Ruiz, F. X., Poirson, J., Mitschler, A., Ramirez, J., Forster, A., et al. (2016). Structure of the E6/E6AP/p53 complex required for HPVmediated degradation of p53. Nature 529, 541-545. doi: 10.1038/nature16481

Maspero, E., Mari, S., Valentini, E., Musacchio, A., Fish, A., Pasqualato, S., et al. (2011). Structure of the HECT:ubiquitin complex and its role in ubiquitin chain elongation. EMBO Rep. 12, 342-349. doi: 10.1038/embor.2011.21

Maspero, E., Valentini, E., Mari, S., Cecatiello, V., Soffientini, P., Pasqualato, S., et al. (2013). Structure of a ubiquitin-loaded HECT ligase reveals the molecular basis for catalytic priming. Nat. Struct. Mol. Biol. 20, 696-701. doi: 10.1038/nsmb. 2566

Metzger, M. B., Pruneda, J. N., Klevit, R. E., and Weissman, A. M. (2014). RINGtype E3 ligases: master manipulators of E2 ubiquitin-conjugating enzymes and ubiquitination. Biochim. Biophys. Acta 1843, 47-60. doi: 10.1016/j.bbamcr.2013. 05.026

Mortensen, F., Schneider, D., Barbic, T., Sladewska-Marquardt, A., Kuhnle, S., Marx, A., et al. (2015). Role of ubiquitin and the HPV E6 oncoprotein in E6AP-mediated ubiquitination. Proc. Natl. Acad. Sci. U.S.A. 112, 9872-9877. doi: 10.1073/pnas.1505923112

Mund, T., Lewis, M. J., Maslen, S., and Pelham, H. R. (2014). Peptide and small molecule inhibitors of HECT-type ubiquitin ligases. Proc. Natl. Acad. Sci. U.S.A. 111, 16736-16741. doi: 10.1073/pnas.1412152111

Mund, T., and Pelham, H. R. (2009). Control of the activity of WW-HECT domain E3 ubiquitin ligases by NDFIP proteins. EMBO Rep. 10, 501-507. doi: 10.1038/ embor. 2009.30
Mund, T., and Pelham, H. R. (2010). Regulation of PTEN/Akt and MAP kinase signaling pathways by the ubiquitin ligase activators Ndfip1 and Ndfip2. Proc. Natl. Acad. Sci. U.S.A. 107, 11429-11434. doi: 10.1073/pnas.0911714107

Myant, K. B., Cammareri, P., Hodder, M. C., Wills, J., Von Kriegsheim, A., Gyorffy, B., et al. (2017). HUWE1 is a critical colonic tumour suppressor gene that prevents MYC signalling, DNA damage accumulation and tumour initiation. EMBO Mol. Med. 9, 181-197. doi: 10.15252/emmm.201606684

Ogunjimi, A. A., Briant, D. J., Pece-Barbara, N., Le Roy, C., Di Guglielmo, G. M., Kavsak, P., et al. (2005). Regulation of Smurf2 ubiquitin ligase activity by anchoring the E2 to the HECT domain. Mol. Cell 19, 297-308. doi: 10.1016/ j.molcel.2005.06.028

Oh, E., Akopian, D., and Rape, M. (2018). Principles of ubiquitin-dependent signaling. Annu. Rev. Cell Dev. Biol. 34, 137-162. doi: 10.1146/annurev-cellbio100617-062802

Okada, M., Sangadala, S., Liu, Y., Yoshida, M., Reddy, B. V., Titus, L., et al. (2009). Development and optimization of a cell-based assay for the selection of synthetic compounds that potentiate bone morphogenetic protein-2 activity. Cell Biochem. Funct. 27, 526-534. doi: 10.1002/cbf.1615

Persaud, A., Alberts, P., Amsen, E. M., Xiong, X., Wasmuth, J., Saadon, Z., et al. (2009). Comparison of substrate specificity of the ubiquitin ligases Nedd4 and Nedd4-2 using proteome arrays. Mol. Syst. Biol. 5:333. doi: $10.1038 / \mathrm{msb} .2009 .85$

Persaud, A., Alberts, P., Mari, S., Tong, J., Murchie, R., Maspero, E., et al. (2014). Tyrosine phosphorylation of NEDD4 activates its ubiquitin ligase activity. Sci. Signal. 7:ra95. doi: 10.1126/scisignal.2005290

Peter, S., Bultinck, J., Myant, K., Jaenicke, L. A., Walz, S., Muller, J., et al. (2014). H Tumor cell-specific inhibition of MYC function using small molecule inhibitors of the HUWE1 ubiquitin ligase. EMBO Mol. Med. 6, 1525-1541. doi: 10.15252/ emmm.201403927

Polo, S., and Di Fiore, P. P. (2008). Finding the right partner: science or ART? Cell 135, 590-592. doi: 10.1016/j.cell.2008.10.032

Quirit, J. G., Lavrenov, S. N., Poindexter, K., Xu, J., Kyauk, C., Durkin, K. A., et al. (2017). Indole-3-carbinol (I3C) analogues are potent small molecule inhibitors of NEDD4-1 ubiquitin ligase activity that disrupt proliferation of human melanoma cells. Biochem. Pharmacol. 127, 13-27. doi: 10.1016/j.bcp. 2016.12.007

Ramamoorthy, S., Tufail, R., Hokayem, J. E., Jorda, M., Zhao, W., Reis, Z., et al. (2012). Overexpression of ligase defective E6-associated protein, E6-AP, results in mammary tumorigenesis. Breast Cancer Res. Treat. 132, 97-108. doi: 10.1007/s10549-011-1567-2

Reiter, K. H., and Klevit, R. E. (2018). Characterization of RING-Between-RING E3 ubiquitin transfer mechanisms. Methods Mol. Biol. 1844, 3-17. doi: 10.1007/ 978-1-4939-8706-1_1

Riling, C., Kamadurai, H., Kumar, S., O’Leary, C. E., Wu, K. P., Manion, E. E., et al. (2015). Itch WW domains inhibit its E3 ubiquitin ligase activity by blocking E2-E3 ligase trans-thiolation. J. Biol. Chem. 290, 23875-23887. doi: 10.1074/jbc. M115.649269

Ronchi, V. P., Klein, J. M., Edwards, D. J., and Haas, A. L. (2014). The active form of E6-associated protein (E6AP)/UBE3A ubiquitin ligase is an oligomer. J. Biol. Chem. 289, 1033-1048. doi: 10.1074/jbc.M113.517805

Rossi, M., Rotblat, B., Ansell, K., Amelio, I., Caraglia, M., Misso, G., et al. (2014). High throughput screening for inhibitors of the HECT ubiquitin E3 ligase ITCH identifies antidepressant drugs as regulators of autophagy. Cell Death Dis. 5:e1203. doi: 10.1038/cddis.2014.113

Rotin, D., and Kumar, S. (2009). Physiological functions of the HECT family of ubiquitin ligases. Nat. Rev. Mol. Cell. Biol. 10, 398-409. doi: 10.1038/nrm2690

Sanchez-Tena, S., Cubillos-Rojas, M., Schneider, T., and Rosa, J. L. (2016). Functional and pathological relevance of HERC family proteins: a decade later. Cell Mol. Life Sci. 73, 1955-1968. doi: 10.1007/s00018-016-2139-8

Sander, B., Xu, W., Eilers, M., Popov, N., and Lorenz, S. (2017). A conformational switch regulates the ubiquitin ligase HUWE1. Elife 6:e21036. doi: 10.7554/eLife. 21036

Scheffner, M., Huibregtse, J. M., Vierstra, R. D., and Howley, P. M. (1993). The HPV-16 E6 and E6-AP complex functions as a ubiquitin-protein ligase in the ubiquitination of p53. Cell 75, 495-505. doi: 10.1016/0092-8674(93)90384-3 
Scheffner, M., and Kumar, S. (2014). Mammalian HECT ubiquitin-protein ligases: biological and pathophysiological aspects. Biochim. Biophys. Acta Mol. Cell Res. 1843, 61-74. doi: 10.1016/j.bbamcr.2013.03.024

Shao, C., Li, Z., Ahmad, N., and Liu, X. (2017). Regulation of PTEN degradation and NEDD4-1 E3 ligase activity by Numb. Cell Cycle 16, 957-967. doi: 10.1080/ 15384101.2017.1310351

Shearwin-Whyatt, L., Dalton, H. E., Foot, N., and Kumar, S. (2006). Regulation of functional diversity within the Nedd 4 family by accessory and adaptor proteins. Bioessays 28, 617-628. doi: 10.1002/bies.20422

Sluimer, J., and Distel, B. (2018). Regulating the human HECT E3 ligases. Cell Mol. Life Sci. 75, 3121-3141. doi: 10.1007/s00018-018-2848-2

Srinivasan, S., and Nawaz, Z. (2011). E3 ubiquitin protein ligase, E6-associated protein (E6-AP) regulates PI3K-Akt signaling and prostate cell growth. Biochim. Biophys. Acta 1809, 119-127. doi: 10.1016/j.bbagrm.2010.08.011

Staub, O., Dho, S., Henry, P., Correa, J., Ishikawa, T., McGlade, J., et al. (1996). WW domains of Nedd4 bind to the proline-rich PY motifs in the epithelial $\mathrm{Na}+$ channel deleted in Liddle's syndrome. EMBO J. 15, 2371-2380. doi: 10. 1002/j.1460-2075.1996.tb00593.x

Trimpert, C., Wesche, D., de Groot, T., Pimentel Rodriguez, M. M., Wong, V., van den Berg, D. T. M., et al. (2017). NDFIP allows NEDD4/NEDD4L-induced AQP2 ubiquitination and degradation. PLoS One 12:e0183774. doi: 10.1371/ journal.pone.0183774

Trotman, L. C., Wang, X., Alimonti, A., Chen, Z., Teruya-Feldstein, J., Yang, H., et al. (2007). Ubiquitination regulates PTEN nuclear import and tumor suppression. Cell 128, 141-156. doi: 10.1016/j.cell.2006.11.040

Vecchione, A., Marchese, A., Henry, P., Rotin, D., and Morrione, A. (2003). The Grb10/Nedd4 complex regulates ligand-induced ubiquitination and stability of the insulin-like growth factor I receptor. Mol. Cell Biol. 23, 3363-3372. doi: 10.1128/MCB.23.9.3363-3372.2003

Verdecia, M. A., Joazeiro, C. A., Wells, N. J., Ferrer, J. L., Bowman, M. E., Hunter, T., et al. (2003). Conformational flexibility underlies ubiquitin ligation mediated by the WWP1 HECT domain E3 ligase. Mol. Cell 11, 249-259. doi: 10.1016/S1097-2765(02)00774-8

Wang, D., Ma, L., Wang, B., Liu, J., and Wei, W. (2017). E3 ubiquitin ligases in cancer and implications for therapies. Cancer Metastasis Rev. 36, 683-702. doi: 10.1007/s10555-017-9703-z

Wang, M., and Pickart, C. M. (2005). Different HECT domain ubiquitin ligases employ distinct mechanisms of polyubiquitin chain synthesis. Embo J. 24, 4324-4333. doi: 10.1038/sj.emboj.7600895

Wang, X., Lu, G., Li, L., Yi, J., Yan, K., Wang, Y., et al. (2014). HUWE1 interacts with BRCA1 and promotes its degradation in the ubiquitin-proteasome pathway. Biochem. Biophys. Res. Commun. 444, 290-295. doi: 10.1016/j.bbrc. 2013.12.053
Wang, X., Trotman, L. C., Koppie, T., Alimonti, A., Chen, Z., Gao, Z., et al. (2007). NEDD4-1 is a proto-oncogenic ubiquitin ligase for PTEN. Cell 128, 129-139. doi: 10.1016/j.cell.2006.11.039

Wiesner, S., Ogunjimi, A. A., Wang, H. R., Rotin, D., Sicheri, F., Wrana, J. L., et al. (2007). Autoinhibition of the HECT-type ubiquitin ligase Smurf2 through its C2 domain. Cell 130, 651-662. doi: 10.1016/j.cell.2007.06.050

Wolyniec, K., Shortt, J., de Stanchina, E., Levav-Cohen, Y., Alsheich-Bartok, O., Louria-Hayon, I., et al. (2012). E6AP ubiquitin ligase regulates PML-induced senescence in Myc-driven lymphomagenesis. Blood 120, 822-832. doi: 10.1182/ blood-2011-10-387647

Zeng, T., Wang, Q., Fu, J., Lin, Q., Bi, J., Ding, W., et al. (2014). Impeded Nedd41-mediated Ras degradation underlies Ras-driven tumorigenesis. Cell Rep. 7, 871-882. doi: 10.1016/j.celrep.2014.03.045

Zhang, W., Wu, K. P., Sartori, M. A., Kamadurai, H. B., Ordureau, A., Jiang, C., et al. (2016). System-wide modulation of HECT E3 ligases with selective ubiquitin variant probes. Mol. Cell 62, 121-136. doi: 10.1016/j.molcel.2016.02. 005

Zhao, X., Heng, J. I., Guardavaccaro, D., Jiang, R., Pagano, M., Guillemot, F., et al. (2008). The HECT-domain ubiquitin ligase Huwe1 controls neural differentiation and proliferation by destabilizing the N-Myc oncoprotein. Nat. Cell Biol. 10, 643-653. doi: 10.1038/ncb 1727

Zhong, Q., Gao, W., Du, F., and Wang, X. (2005). Mule/ARF-BP1, a BH3-only E3 ubiquitin ligase, catalyzes the polyubiquitination of $\mathrm{Mcl}-1$ and regulates apoptosis. Cell 121, 1085-1095. doi: 10.1016/j.cell.2005.06.009

Zhu, K., Shan, Z., Chen, X., Cai, Y., Cui, L., Yao, W., et al. (2017). Allosteric autoinhibition and activation of the Nedd4 family E3 ligase Itch. EMBO Rep. 18, 1618-1630. doi: 10.15252/embr.201744454

Zou, X., Levy-Cohen, G., and Blank, M. (2015). Molecular functions of NEDD4 E3 ubiquitin ligases in cancer. Biochim. Biophys. Acta 1856, 91-106. doi: 10.1016/j.bbcan.2015.06.005 doi: 10.1016/j.bbcan.2015. 06.005

Conflict of Interest Statement: The authors declare that the research was conducted in the absence of any commercial or financial relationships that could be construed as a potential conflict of interest.

Copyright (C) 2019 Weber, Polo and Maspero. This is an open-access article distributed under the terms of the Creative Commons Attribution License (CC BY). The use, distribution or reproduction in other forums is permitted, provided the original author(s) and the copyright owner(s) are credited and that the original publication in this journal is cited, in accordance with accepted academic practice. No use, distribution or reproduction is permitted which does not comply with these terms. 